\title{
Microstructural Details and Polymorphs in Poly(propylene-co-1-nonene) Copolymers Synthesized at Different Polymerization Temperatures
}

\author{
Alberto García-Peñas ${ }^{1,2^{*}}$, José M. Gómez-Elvira ${ }^{1}$ Enrique Blázquez-Blázquez¹, Rosa \\ Barranco-García ${ }^{1}$, Ernesto Pérez ${ }^{1 *}$, María L. Cerrada1
}

${ }^{1}$ Instituto de Ciencia y Tecnología de Polímeros (ICTP-CSIC), Juan de la Cierva 3, 28006 Madrid, Spain. ${ }^{2}$ Current address: Universidad Carlos III de Madrid, Avda. de la Universidad, 30 (Edificio Betancourt). 28911, Leganés (Madrid), Spain.

Corresponding authors: E. Pérez (ernestop@ictp.csic.es)

and A. García-Peñas (alberto.garcia.penas@uc3m.es)

\begin{abstract}
Several copolymers based on propylene with 1-nonene as comonomer have been synthesized within a wide range of compositions using a metallocene catalyst and different temperatures. The effect of the two main variables, comonomer content and polymerization temperature, on the final microstructure obtained (polymerization activity, stereochemistry and molar mass, among other details) as well as on the capability of crystallization, formation of distinct polymorphs and phase transitions, is examined. Different ordered entities are developed in these isotactic poly(propylene-co-1-nonene) copolymers depending on 1-nonene molar content: monoclinic, orthorhombic and mesomorphic ones. A detailed analysis of the phase transitions involved is performed using real-time variable-temperature wide angle diffraction experiments with synchrotron radiation on the sample with the highest comonomer amount. Different crystallization conditions were tested in order to attain information of the whole phase behavior. From these results, it was found that the mesophase of iPP is competing with the crystalline structures, although it recrystallizes on melting into the monoclinic phase at common DSC rates.
\end{abstract}

\section{Keywords}

Polypropylene; 1-nonene; polymerization temperature; microstructure; mesophase; orthorhombic phase; monoclinic phase. 


\section{Introduction}

Manufacture of polyolefins, including polyethylene (PE) and polypropylene (PP), involves actually more than half of commercial polymers worldwide. They are mostly employed in commodity applications that affect our daily life, because of their unique combination of chemical and physical properties, excellent processability and recyclability, together with their low cost. Nevertheless, scenario is changing in relation to those commodity uses due to new environmental policies and regulations. Important efforts are focused on improvements in the reprocessing of polyolefin wastes or their recovery as chemical resources. ${ }^{[1-5]}$

The advances on catalytic systems, processes optimization and environmental friendly routes could offer interesting opportunities to deal with new regulations.[[6,7] In this context, polymerization parameters together with the type of catalyst used have shown that can deeply affect the final molecular features and, consequently, the ultimate product performance. ${ }^{[8,9]}$ In the early $1980 \mathrm{~s}$, homogeneous metallocene catalysts triggered a new era in the polyolefin academia and industry as well as an opportunity to develop novel polyolefin structures.[10-14] In addition to their superior catalytic activities, the major advantage of the metallocene catalysts was their single, well-defined, and tunable active sites (by changing ligands) and, consequently, its well-controlled reaction mechanism. Several PE and PP copolymers containing large comonomers, including alpha-olefinic, styrenic and cyclic ones, were effectively prepared with high molecular weight, a broad range of copolymer compositions, and narrow molecular weight and composition distributions.

Those synthetic achievements, allowing both random distributions and large incorporations in comonomer, led to architectures based on isotactic polypropylene that exhibited a new polymorph (in addition to the well known iPP polymorphs, detailed below). It was described in 2005 for the first time in literature for random copolymers of propylene and 1-hexene with high content in comonomer, ${ }^{[15]}$ above $10 \mathrm{~mol} \%$. This novel crystalline lattice was identified as a trigonal modification ${ }^{[16-18]}$ and was also developed in copolymers of propylene and 1-pentene ${ }^{[19-22]}$ as well as in terpolymers of propylene with 1pentene and 1-hexene ${ }^{[23-25]}$ as comonomers or in terpolymers of propylene with 1-pentene and 1heptene, ${ }^{[26]}$ all of them with high content in comonomers. Nevertheless, this trigonal lattice was not developed in copolymers of propylene and 1-heptene ${ }^{[27,28]}$ or in other ones with longer comonomers (1octene, 1-dodecene, 1-octadecene, among others).[29-31] It was also concluded that this polymorph could not crystallize in polypropylene homopolymer because it would imply too low density.[16]

Additionally, a new crystal modification, named as $\varepsilon$, has been reported in a sample of highly stereodefective ${ }^{[32]}$ isotactic polypropylene, obtained with a zirconocene catalyst. 
Copolymerization of propylene has traditionally been explored using ethylene, 1-butene or 1hexene as commoners of choice. ${ }^{[33-36]}$ However, the use of olefins with an odd number of carbons has

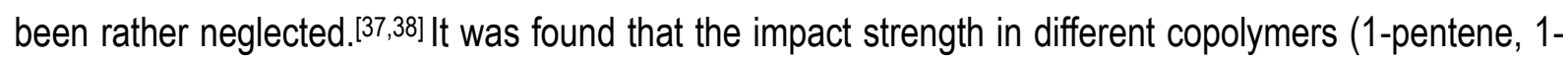
heptene and 1-nonene) is dependent, as expected, on comonomer content, but also on its length. Those incorporating 1-nonene as counit show higher values of impact strength, at smaller comonomer compositions, than those exhibited by their homologues with 1-pentene and 1-heptene. Moreover, it was also found in propylene copolymers that impact strength does not start increasing immediately, as observed for the ethylene copolymers, ${ }^{[39]}$ but the rise is more gradual and follows an exponential increase with comonomer content.[38] More recently, the interest in the copolymers or terpolymers of propylene with 1-pentene or 1-heptene was concentrated primarily on the ability of developing the new trigonal crystalline lattice, as mentioned above, ${ }^{[19-22,27,28]}$ but there are not systematic studies on microstructure and crystalline characteristics for copolymers with 1-nonene.

Our group has lately focused on the synthesis of propylene architectures (either homopolymers or incorporating different a-olefins) using metallocene catalysts to learn on polymerization processes and to further evaluate crystalline characteristics and mechanical properties in the resultant propylene based materials. These polymerizations were performed using homogenous $[21,22,24,26,27]$ or supported[40] catalytic systems.

In order to find some added value and to look for future perspectives of polypropylene materials, the aim of this research is to get a deeper knowledge on copolymers of propylene and 1-nonene, based on some promising properties reported on literature. [38] Thus, their synthesis has been carried out by varying two very important polymerization characteristics: temperature and comonomer content. These two variables have been selected as control parameters in an attempt to assess changes in the final microstructure, which has been thoroughly analyzed by nuclear magnetic resonance (NMR) technique. The influence of those molecular features on the final polymorphs and their thermal transitions has also been preliminary evaluated by wide-angle X-ray diffraction (WAXD) with either conventional or synchrotron radiation and differential scanning calorimetry (DSC).

\section{Experimental}

\section{Materials}

Reagents and solvents were purified and stored under an inert atmosphere of nitrogen. Toluene (Merck) and 1-nonene comonomer (TCl Tokyo Kasei) have been distilled over sodium to avoid presence of traces containing water and/or oxygen. Both propylene (Praxair 2.5) and nitrogen (Conptse) were purified using oxygen-trap columns and molecular sieves. Catalyst, the rac-dimethylsilylbis(1- 
indenyl) zirconium dichloride (Strem), and cocatalyst, the modified methylaluminoxane (MMAO) solution in toluene (7 wt.\% solution in toluene, from Aldrich), were used as received. The activated catalytic system was prepared by dissolving $15 \mathrm{mg}$ of the metallocene complex in $3 \mathrm{~mL}$ of MMAO solution, and then, a volume of $0.313 \mathrm{~mL}$ was used for each polymerization. Ethanol (Aroca, 96\%) and $\mathrm{HCl}$ (VWR, $37 \%$ ) were used for precipitation of the copolymers.

Synthesis of poly(propylene-co-1-nonene) copolymers

The copolymers of propylene and 1-nonene were synthesized in a Büchi glass ecoclave using $250 \mathrm{~mL}$ of toluene. Two sets of copolymers were prepared, selecting reaction temperature and comonomer content as polymerization parameters. In the first one, the polymerization temperature was $-5{ }^{\circ} \mathrm{C}$, with varying the 1-nonene feeding. Different polymerization temperatures were chosen in the second group, with a single value of initial monomers ratio. A homopolymer iPP sample was also synthesized at $-5^{\circ} \mathrm{C}$.

The reaction started with the incorporation of rac-dimethylsilylbis(1-indenyl) zirconium dichloride and MMAO as catalyst/cocatalyst system, and was stopped adding ethanol (around $5 \mathrm{~mL}$ ). The precipitated powders were isolated from reactor by pouring the reaction batch on a solution of ethanol and chloride acid (30:1). The mixtures were stirred overnight, filtrated and washed again with ethanol. Finally, the polymeric materials were dried under vacuum at room temperature.

Table 1 shows the different copolymers attained, which are referred as CPN followed by the value of the 1-nonene mol \% content in the copolymer for the first set of copolymers, where the polymerization temperature was $-5^{\circ} \mathrm{C}$, as indicated above. For the second group, the polymerization temperature is also included, which is indicated by $T$ and its corresponding value.

Film samples of the different copolymers (and of the homopolymer) were obtained by compression molding in a Collin press between hot plates (at a temperature of $30^{\circ} \mathrm{C}$ above the melting point) with a pressure of $2.5 \mathrm{MPa}$ for $3 \mathrm{~min}$, and subsequently cooled down to room temperature at the inherent cooling rate of the press, after the power was switched off (the cooling rate was around 0.5$1{ }^{\circ} \mathrm{C} / \mathrm{min}$, the higher limit for iPP and the lower one, for copolymer cPN7.9T60). Pressure was maintained constant at $2.5 \mathrm{MPa}$ along cooling treatment.

Size exclusion chromatography

The molecular weights were estimated by size exclusion chromatography (SEC) in a Waters GPC/V 2000 (this equipment includes detectors for refractive index and viscosimetry). Three columns of PL Gel type (3 PL Gel 20 mm Mixed A) were employed, and 1,2,4-trichlorobenzene was selected as 
solvent, working at $145{ }^{\circ} \mathrm{C}$ and a flow rate of $1 \mathrm{~mL} / \mathrm{min}$ for the experiments. Previously, the machine was calibrated with diverse standards of polystyrene with narrow molecular mass distribution.

\section{Nuclear magnetic resonance}

The comonomer content, compositional triads and tacticity were estimated by carbon-13 nuclear magnetic resonance analysis, ${ }^{13} \mathrm{C}$ NMR, through an Innova 400 spectrometer (100 MHz). The measurements were carried out from polymeric solutions in 1,1,2,2-tetrachloroethane- $\mathrm{d}_{4}(70 \mathrm{mg} / 1 \mathrm{~mL})$ at $80{ }^{\circ} \mathrm{C}$ using an acquisition time of $1 \mathrm{~s}$, a relaxation delay of $4 \mathrm{~s}$ and a pulse angle of $45^{\circ}$. Thus, 8000 scans were recorded with broad band proton decoupling.

$X$-ray diffraction and synchrotron experiments

Wide-angle X-Ray diffraction (WAXD) patterns were recorded in the reflection mode by using a Bruker D8 Advance diffractometer provided with a PSD Vantec detector (from Bruker, Madison, Wisconsin). Cu Ka radiation ( $\lambda=0.1542 \mathrm{~nm}$ ) was used, operating at $40 \mathrm{kV}$ and $40 \mathrm{~mA}$. The parallel beam optics was adjusted by a parabolic Göbel mirror with horizontal grazing incidence Soller slit of $0.12^{\circ}$ and LiF monochromator. The equipment was calibrated with different standards: $\mathrm{Al}_{2} \mathrm{O}_{3}$ (Corundum) and $\mathrm{Cr}_{2} \mathrm{O}_{3}$. A step scanning mode was employed for the detector. The diffraction scans were collected with a $2 \theta$ step of $0.024^{\circ}$ and 0.2 s per step.

Real-time variable-temperature WAXD experiments with synchrotron radiation were performed at beamline BL11-NCD-SWEET at ALBA (Cerdanyola del Vallés, Barcelona, Spain) at a fixed wavelength of $0.1 \mathrm{~nm}$. The profiles were acquired with a Rayonix LX255-HS detector, placed at about $16 \mathrm{~cm}$ from sample and a tilt angle of around 30 degrees. The temperature control unit was a Linkam hot stage, connected to a cooling system working with liquid nitrogen. For practical purposes, the lowest temperature in these experiments was set to $-20^{\circ} \mathrm{C}$.

The calibration of spacings was obtained by means of silver behenate and $\mathrm{Cr}_{2} \mathrm{O}_{3}$ standards. The initial 2D X-ray images were converted into 1D diffractograms, as function of the inverse scattering vector, $s=1 / d=2 \sin \theta / \lambda$, by means of pyFAI python code (ESRF), modified by ALBA beamline staff.

Differential scanning calorimetry

The calorimetric analysis was performed in a TA Q100 calorimeter connected to a cooling system. The equipment was calibrated with different standards (indium, zinc and $n$-dodecane). Sample weights ranged from 2 to $3 \mathrm{mg}$. A scanning rate of $10^{\circ} \mathrm{C} / \mathrm{min}$ was applied for the different runs. 


\section{Results and discussion}

Table 1 lists some important results derived from the different polymerization runs, such as comonomer ratio, apparent activity and the corresponding yields. Assessment of apparent activities allows understanding several molecular features for the different copolymers obtained. These are represented in Figure 1. Distinct trends can be observed depending on 1-nonene ratio (left plot) and polymerization temperature (right plot).

Table 1. Data from the different copolymer synthesized at several temperatures attained in the polymerization runs: temperature ( $\left.\mathrm{T}_{\text {pol }}\right)$, global feed ratio in 1-nonene ([C9]/([C3]+[C9]), apparent activity and yield. Comonomer content (mol \%) determined from ${ }^{13} \mathrm{C}$ NMR; and average molecular weight $\left(\mathrm{M}_{\mathrm{w}}\right)$ and polydispersity (PDI) deduced from SEC experiments.

\begin{tabular}{cccccccc}
\hline Name & $\begin{array}{c}\mathrm{T}_{\mathrm{pol}} \\
\left({ }^{\circ} \mathrm{C}\right)\end{array}$ & $[\mathrm{C} 9] /(\mathrm{C} 3]+[\mathrm{C} 9])$ & $\begin{array}{c}\text { Activity } \\
\left(\mathrm{kg} \mathrm{mol}^{-1}(\mathrm{prop})\right. \\
\left.\mathrm{mol}^{-1}(\mathrm{cat}) \mathrm{h}^{-1}\right)\end{array}$ & $\begin{array}{c}\text { Yield } \\
(\mathrm{g})\end{array}$ & $\begin{array}{c}\text { Comonomer } \\
\text { content } \\
(\mathrm{mol} \%)\end{array}$ & $\begin{array}{c}\mathrm{M}_{\mathrm{w}} \\
(\mathrm{g} / \mathrm{mol})\end{array}$ & PDI \\
\hline iPP & -5 & 0 & 440 & - & 0 & 140,900 & 2.3 \\
\hline cPN2.0 & -5 & - & - & - & 2.0 & - &. \\
\hline cPN2.8 & -5 & 0.042 & 640 & 1.01 & 2.8 & 137,000 & 3.3 \\
cPN4.2 & -5 & 0.062 & 2,880 & 1.55 & 4.2 & 122,350 & 3.4 \\
CPN5.8 & -5 & 0.080 & 3,400 & 2.21 & 4.8 & 116,500 & 3.1 \\
cPN6.2 & -5 & 0.099 & 1,870 & 1.31 & 6.2 & 98,800 & 2.9 \\
\hline cPN4.0T10 & 10 & 0.042 & 1,270 & 0.94 & 4.0 & 86,100 & 3.1 \\
cPN5.1T25 & 25 & 0.042 & 19,790 & 1.67 & 5.1 & 66,400 & 2.7 \\
cPN7.4T40 & 40 & 0.042 & 55,480 & 4.91 & 7.4 & 30,500 & 2.5 \\
cPN7.9T60 & 60 & 0.042 & 69,600 & 1.53 & 7.9 & 25,150 & 2.4 \\
\hline
\end{tabular}

Figure 1(a) shows that apparent activity increases as the 1-nonene feeding does but it goes through a maximum value, observed at around 0.07 , and then activity decreases. It was reported in literature that the increase in the apparent activities is due to different factors ${ }^{[41-50]}$ associated with an improved solubility of macrochains in the medium, a greater chain transfer activity and the modification of the active center quality. A similar tendency was described in propylene terpolymers with 1-pentene and 1-hexene as comonomers. ${ }^{[51]}$ Apparent activity was also analyzed in the neat propylene-1-pentene (cPPe) and propylene-1-hexene (cPHe) copolymers. It was observed that the one for cPPe was somewhat higher than that for $\mathrm{cPHe}$ copolymers but this variation with comonomer length could not be confirmed by the terpolymerization data through the appearance of two trends depending on weather 
the feeding was richer in the 1-pentene or 1-hexene counits, because differences were not significant enough. Values found in these cPN copolymers are lower than those reported in $\mathrm{cPPe}$ and $\mathrm{cPHe}$ copolymers, what seems to indicate that apparent activity decreases as comonomer length increases.

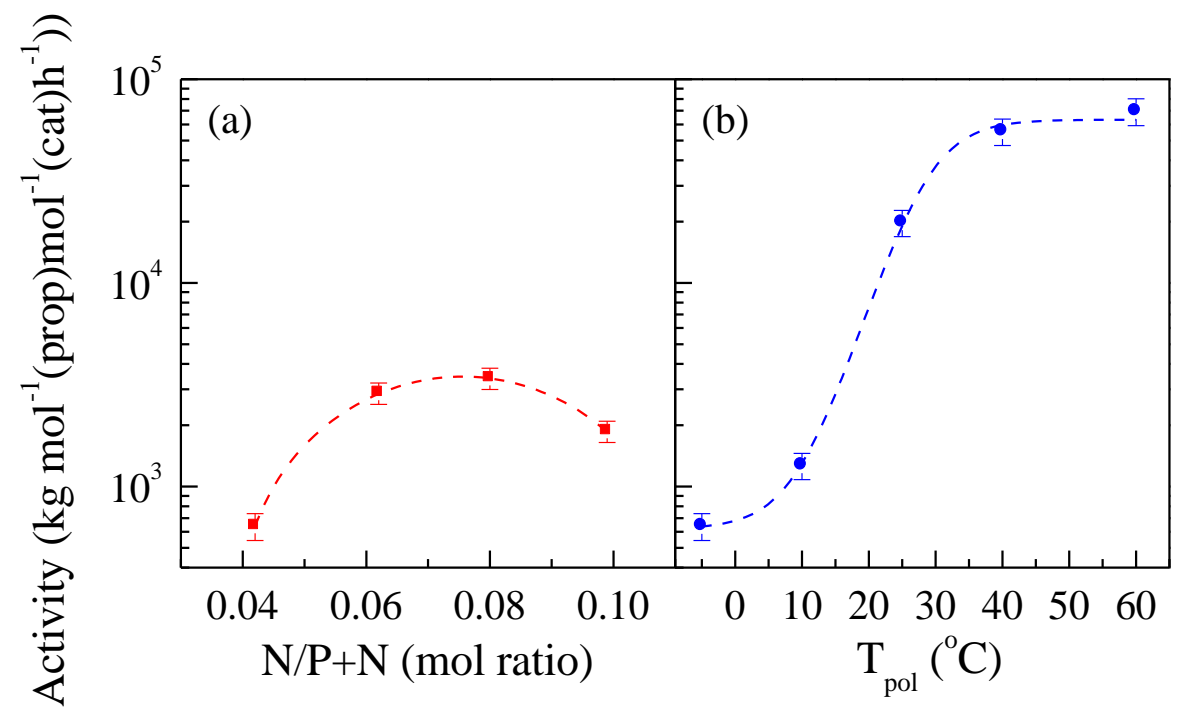

Figure 1. Apparent catalytic activity as function of: (a) global feed ratio in 1-nonene; and, (b) polymerization temperatures.

The effect of polymerization temperature is rather important on the apparent activity, which rises as temperature is increased. This influence was previously described and associated with a higher number of active centers inside the reactor. ${ }^{[52]}$ Plot (b) in Figure 1 depicts that these cPN copolymers show a sigmoid behavior. Thus, activity is rather similar for the highest temperatures, indicating a slowdown probably due to hindrance imposed between polymeric chains. ${ }^{[53]}$

Average molecular weight is another important magnitude that is significantly affected by comonomer content and polymerization temperatures, as listed in Table 1. Various changes associated with the existence of chain transfer reactions can take place as 1-nonene content rises in the reaction medium. Consequently, a decrease in molecular weight is observed in the set of copolymers synthesized at $-5^{\circ} \mathrm{C}$ as comonomer content increases. This behavior has been extensively reported in the literature. $[41,42,50]$

The effect of temperature on molar mass is also well known. An increase of temperature leads to a rise of active centers that will promote a large number of polymeric chains in growth, but also a higher extent of termination events. ${ }^{[54]}$ Table 1 shows the considerable difference found between cPN4.2 and CPN4.0T10, both with similar comonomer content. The molecular weight $\mathrm{M}_{\mathrm{w}}$ is reduced from 122,350 to 86,100 , respectively, indicating a decrease of about $36,000 \mathrm{~g} / \mathrm{mol}$ for the sample polymerized at the highest reaction temperature. 
Comonomer content, estimated by ${ }^{13} \mathrm{C}$ NMR, for the different copolymers is also displayed in Table 1. Incorporation of 1-nonene into polypropylenic chains seems to be favored when polymerization temperature is increased, as can be deduced from the results in Table 1. In fact, analogous 1-nonene feeding was used for preparing cPN2.8, cPN4.0, cPN5.1, cPN7.4 and cPN7.9, at -5, 10, 25, 40 and $60{ }^{\circ} \mathrm{C}$, respectively, although the final 1-nonene incorporations reached were different, as can be deduced from comonomer amount reported in Table 1. This effect can be explained by the reaction temperature phenomenon. A polymerization performed at a higher temperature leads to an increase of the pressure inside the reactor and, consequently, the concentration of propylene incorporated is noticeably lowered since the propylene amount within the reactor is triggered by pressure. Thus, polymerization temperature plays an important role for ruling the content of 1-nonene. Its precise control could be a useful tool for optimizing the production of these copolymers and the saving of an important amount of raw materials.

Temperature does not only affect the final 1-nonene incorporation, as just commented, but also influences the resultant molar mass (see values in Table 1) as well as the microstructure, mainly isotacticity (see below). The existence of secondary reactions, like transfer reactions, or the deactivation of active centers is behind these variations. Anyway, it was reported that controlled polymerization using metallocene catalysts follows the Schulz-Flory distribution.[55]

To elucidate changes in microstructure, assignment of the various carbons associated with both propylene and 1-nonene inserted into the polymeric chains has been performed from their characteristic peaks observed in the ${ }^{13} \mathrm{C}$ NMR spectra. They are identified in Figure 2 and labeled regarding chemical shifts specified in Table 2. The signals are assigned in accordance with data reported in the literature related to isotactic polypropylene, poly(1-heptene) and poly(1-nonene).[56] The carbons are named as $P$ and $\mathrm{N}$ as belonging to propylene and 1-nonene comonomers, respectively. The assignment is clearly defined in Scheme 1, the superscript denoting position of the diverse carbons.

Scheme 1. Carbon assignment belonging to propylene $(P)$ and 1-nonene $(N)$ in the polymeric chain.

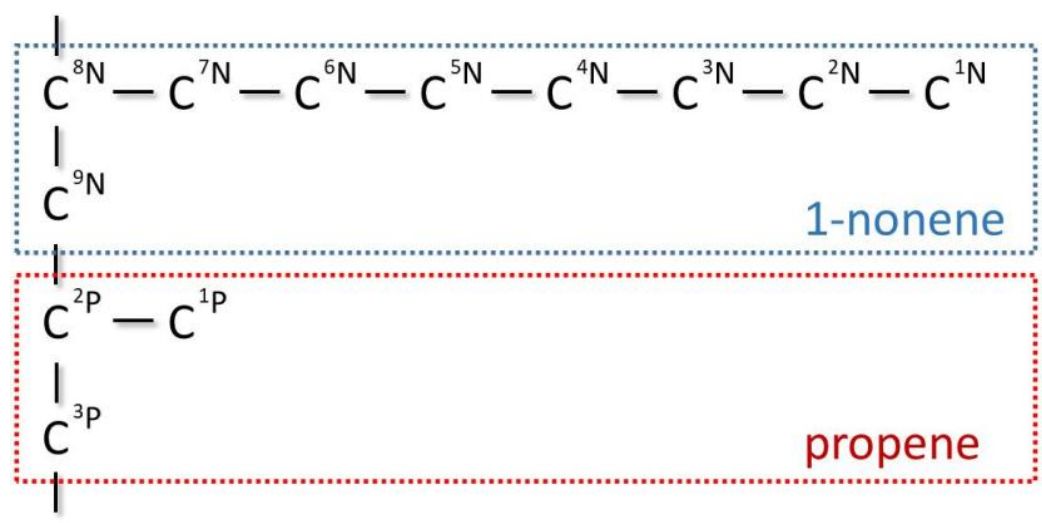




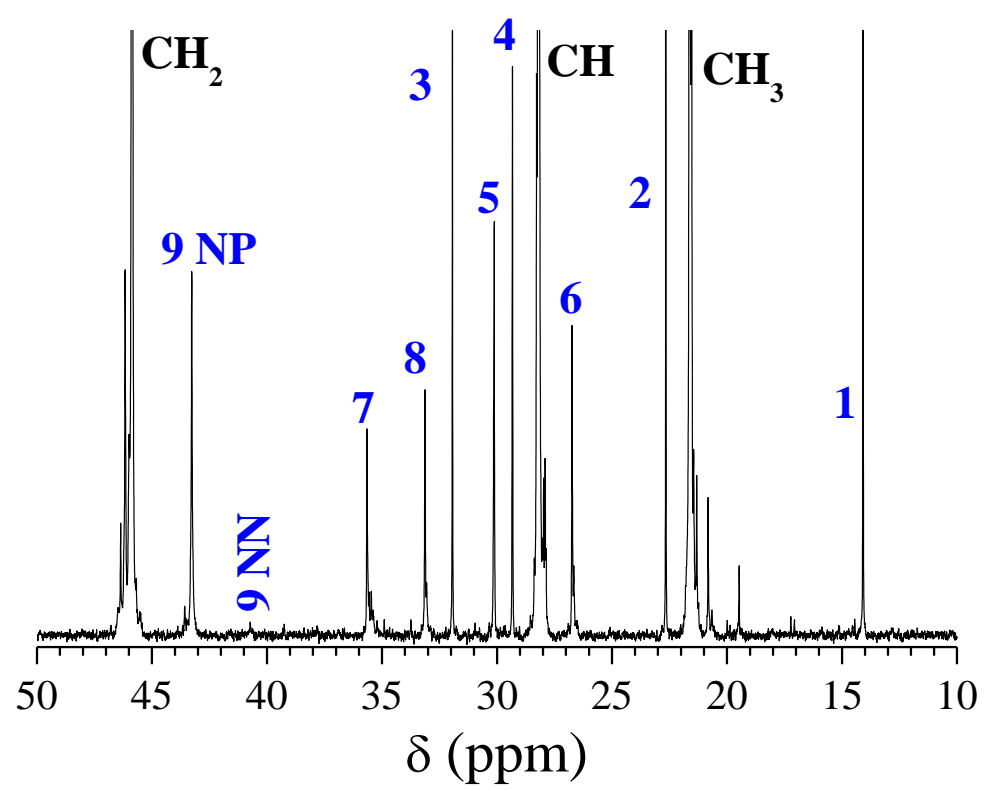

Figure 2. Identification of the different carbon nuclei in the ${ }^{13} \mathrm{C}$ NMR spectrum for CPN7.4T40 obtained in 1,1,2,2-tetrachloroethane- $d_{4}$ at $80^{\circ} \mathrm{C}$.

Table 2. Carbon assignments and experimental chemical shifts from ${ }^{13} \mathrm{C}$ NMR spectra for poly(propeneco-1-nonene) copolymers according to Scheme 1.

\begin{tabular}{|c|c|c|}
\hline Peak & $\begin{array}{c}\text { Carbon nucleus: } \\
(\mathrm{P}=\text { propylene } ; \mathrm{N}=1 \text {-nonene })\end{array}$ & $\begin{array}{l}\text { Chemical shift } \\
\qquad(p p m)^{a}\end{array}$ \\
\hline 1 & ${ }^{1} \mathrm{~N}$ & 14.09 \\
\hline $\mathrm{CH}_{3}$ & ${ }^{1} P\left(P^{1} P P+P^{1} P N\right)$ & 21.63 \\
\hline 2 & $2 \mathrm{~N}$ & 22.63 \\
\hline 6 & $6 \mathrm{~N}$ & 26.76 \\
\hline $\mathrm{CH}$ & ${ }^{2} \mathrm{P}\left(\mathrm{P}^{2} \mathrm{PP}+\mathrm{P}^{2} \mathrm{PH}\right)$ & 28.19 \\
\hline 4 & $4 \mathrm{~N}$ & 29.32 \\
\hline 5 & ${ }^{5} \mathrm{~N}$ & 30.10 \\
\hline 3 & $3 \mathrm{~N}$ & 31.96 \\
\hline 8 & $8 \mathrm{~N}$ & 33.09 \\
\hline 7 & $7 \mathrm{~N}$ & 35.66 \\
\hline \multirow{2}{*}{9} & PN9NP & 40.64 \\
\hline & $9 \mathrm{~N}$ & 43.27 \\
\hline \multirow{2}{*}{$\mathrm{CH}_{2}$} & PP3PP & 45.91 \\
\hline & PP3PN & 46.15 \\
\hline
\end{tabular}

a Chemical shifts are referenced to $\mathrm{C}_{4} \mathrm{D}_{2} \mathrm{Cl}_{4}(74.00 \mathrm{ppm})$. 
Figure 3 shows the different propylene tacticity sequences observed from the methyl region of ${ }^{13} \mathrm{C}$ NMR spectra at pentad level. Then, the tacticity distribution is estimated and presented in Table 3. On one hand, $\mathrm{mmmm}$ pentads decrease as 1-nonene content is incorporated into polymeric chains in the copolymers prepared at $-5{ }^{\circ} \mathrm{C}$, with the exception of $\mathrm{cPN} 2.8$, as previously reported for similar compositions. ${ }^{24]}$ Moreover, isotacticity decreases dramatically as polymerization temperature is raised. On the other hand, mmmr sequences are enlarged as comonomer content rises and also, significantly, as polymerization temperature increases. The growth in $\mathrm{mmmr}$ sequences could be explained from the temperature effect on frequency of error insertions, ${ }^{[57]}$ as can be seen from the ${ }^{13} \mathrm{C}$ NMR peaks. Nevertheless, the appearance of other tacticity sequences could be associated with other effects. ${ }^{\left[{ }^{[1]}\right.}$

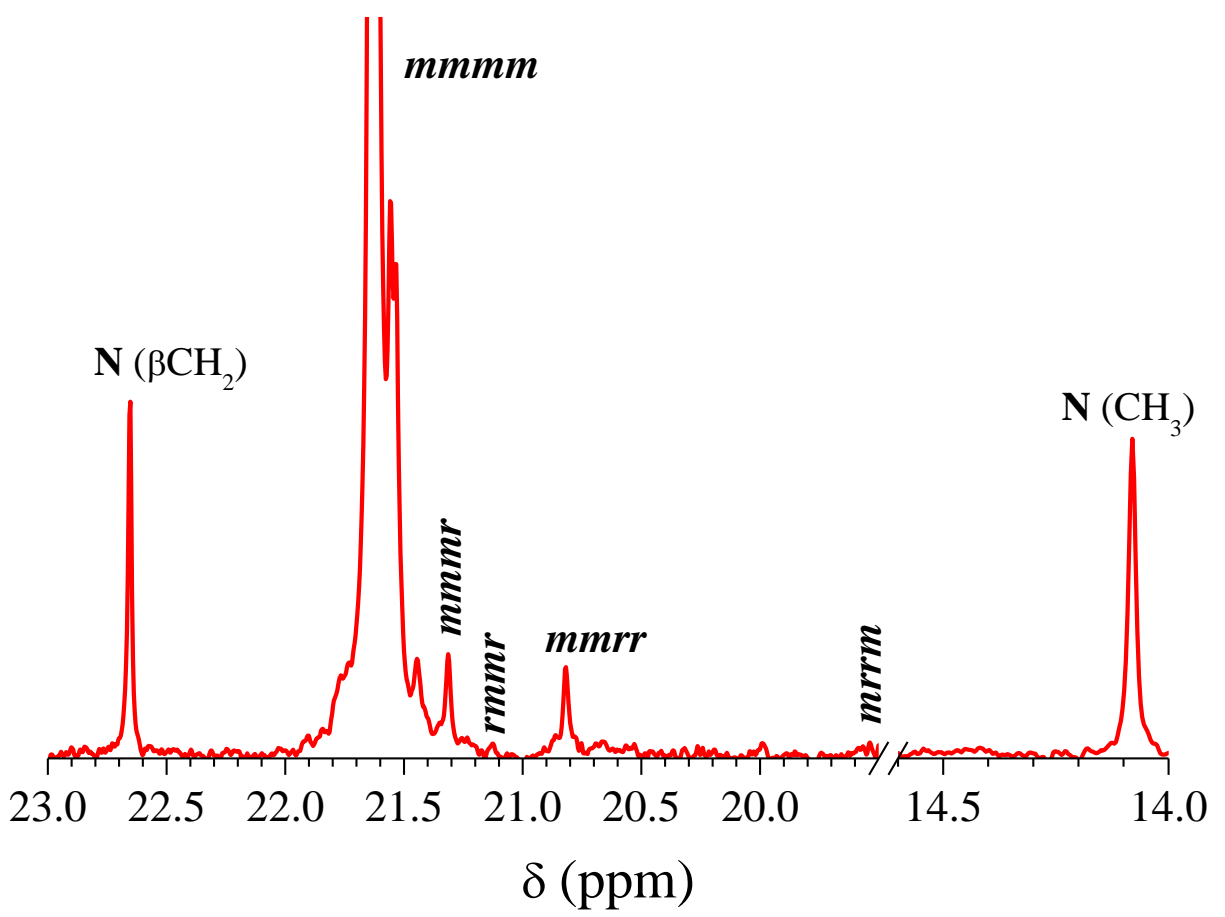

Figure 3. Methyl ${ }^{13} \mathrm{C}$ NMR spectral window for the cPN2.8 copolymer obtained in 1,1,2,2tetrachloroethane- $d_{4}$ at $80^{\circ} \mathrm{C}$.

The metallocene catalyst used in these polymerizations leads only to obtaining $\mathrm{mrrm}$ sequences as stereodefects. These sequences rise clearly with increasing reaction temperature. Additional information can be deduced from Figure 4 where the main tacticity pentads are represented as a function of polymerization temperature. In general, $m m m r, r m m r, m m r r$ and $m r r m$ sequences increase exponentially as temperature does. It is well known that catalytic activity triggers the final microstructure within polymeric macrochains.[58] Consequently, polymerization temperature and incorporation of comonomer exert a deep effect on the final tacticity exhibited by the resulting copolymers. 
Table 3. Propylene tacticity at the pentad level for the different copolymers analyzed.

\begin{tabular}{|c|c|c|c|c|c|c|c|c|c|c|}
\hline Samples & $\stackrel{\text { हิ }}{\text { हิ }}$ & $\underset{\text { हे }}{\grave{\text { ह }}}$ & हे & हे & $\begin{array}{c}m m r m \\
+ \\
\text { rmrr }\end{array}$ & હे & $\Sigma$ & $\stackrel{\vdots}{\Xi}$ & $\underline{\mathbf{s}}$ & $\begin{array}{l}\frac{y}{d} \\
\frac{d}{d} \\
\frac{0}{0} \\
\frac{0}{d} \\
\frac{0}{\alpha}\end{array}$ \\
\hline cPN2.8 & 93.9 & 2.7 & 0 & 2.0 & 0 & 0 & 0 & 0 & 0.8 & 0.6 \\
\hline cPN4.2 & 94.3 & 3.0 & 0 & 1.8 & 0 & 0 & 0 & 0 & 0.5 & 0.4 \\
\hline cPN4.8 & 94.3 & 2.5 & 0.3 & 1.8 & 0 & 0 & 0 & 0 & 0.7 & 0.4 \\
\hline cPN6.2 & - & - & - & - & - & - & - & - & - & - \\
\hline cPN4.0T10 & 92.6 & 2.9 & 0.2 & 2.2 & 1.1 & 0 & 0 & 0 & 0.7 & 0.3 \\
\hline cPN5.1T25 & 90.3 & 3.7 & 0.9 & 2.7 & 1.0 & 0 & 0 & 0 & 0.9 & 0.5 \\
\hline cPN7.4T40 & 88.5 & 4.4 & 0.8 & 3.2 & 1.3 & 0 & 0 & 0 & 1.3 & 0.5 \\
\hline cPN7.9T60 & 82.5 & 7.4 & 0 & 4.9 & 2.7 & 0 & 0 & 0 & 2.0 & 0.5 \\
\hline
\end{tabular}

The $\mathrm{mmmm}$ pentads decrease around $10 \%$ with increasing polymerization temperature, as deduced when comparing cPN2.8 with cPN7.9T60. However, copolymers prepared at $-5{ }^{\circ} \mathrm{C}$ with varying feeding ratio exhibit similar content in $\mathrm{mmmm}$ pentads, as listed in Table 3 . The effect of reaction temperature is quite evident from the trends described by the $m m m r$ and $m m r r$ sequences, showing an exponential growth, as depicted in Figure 4. The variation on temperature for these distinct pentads clearly indicates that polymerization temperature primarily affects the $\mathrm{mmmm}$ and $\mathrm{mmmr}$. Thus, intervals go from around 94 to $82 \%$ and from about 3 to $7.5 \%$, for the former and latest, respectively. The presence of regiodefects is also observed, which are mainly associated with the homopolymer and copolymers with low comonomer content. ${ }^{[57]}$

Comonomer distribution at the triad level as well as the average propylene length $\left(n_{\mathrm{p}}\right)$ are listed in Table 4 and have been also estimated using the appropriate relationships between ${ }^{13} \mathrm{C}$ NMR integrals, as previously reported.[51] The sequences are not longer than 2 units because the composition in 1nonene is relatively low (below $8 \mathrm{~mol} \%$ in all cases analyzed). Thus, NNN triads are not detected in any of the materials discussed in the present work.

Comonomer is exclusively incorporated as isolated 1-nonene units in cPN2.8, cPN4.2, cPN6.2 and CPN4.0T10, whereas N diads are also exhibited in other copolymers. In general, presence of diads is reported for comonomer compositions higher than $5 \mathrm{~mol} \%$, and they coexist with isolated units that are disrupting the polypropylene segments. ${ }^{[47,59-61]}$ The NNP triads found are below $2.1 \%$, which is an 
expected value since composition range is lower than $8 \mathrm{~mol} \%$. Finally, some NPN triads are also observed for the copolymers whose comonomer content is high enough.

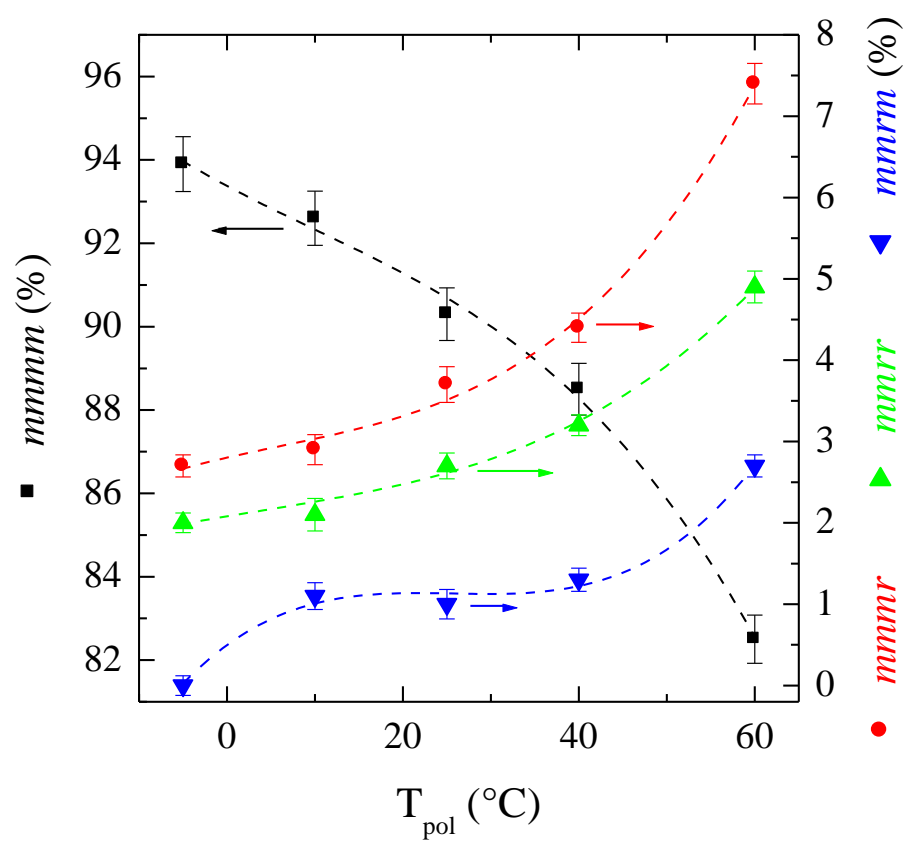

Figure 4. Propylene tacticity for diverse sequences at the pentad level $(\mathrm{mmmm}, \mathrm{mmmr}, \mathrm{mmrr}$ and $\mathrm{mmrm}$ ) for the different copolymers as a function of polymerization temperature.

Table 4. Relative content in triads and average propylene length $\left(n_{\mathrm{p}}\right)$.

\begin{tabular}{cccccccc}
\hline Name & PPP & PPN & NPN & PNP & NNP & NNN & $n_{p}$ \\
\hline cPN2.8 & 91.8 & 5.5 & 0 & 2.7 & 0 & 0 & 35 \\
cPN4.2 & 87.4 & 8.4 & 0 & 4.2 & 0 & 0 & 23 \\
CPN4.8 & 86.2 & 8.1 & 0.4 & 5 & 0.3 & 0 & 21 \\
CPN6.2 & 79.7 & 14.5 & 0 & 5.8 & 0 & 0 & 13 \\
\hline cPN4.0T10 & 87.7 & 7.7 & 0 & 4.6 & 0 & 0 & 25 \\
cPN5.1T25 & 84.8 & 9 & 0.7 & 4.8 & 0.7 & 0 & 18 \\
cPN7.4T40 & 79.5 & 11.6 & 0.9 & 5.9 & 2.1 & 0 & 14 \\
cPN7.9T60 & 78.9 & 11.4 & 1.2 & 6.6 & 1.9 & 0 & 13 \\
\hline
\end{tabular}

The average propylene length $\left(n_{\mathrm{p}}\right)$ decreases clearly as comonomer content increases. The highest $n_{\mathrm{p}}$ value among the analyzed samples is observed for cPN2.8, while the lowest is detected for 
cPN7.9T60. Anyway, it appears that polymerization temperature plays an important role promoting the incorporation of the comonomeric chains.

The compositional triads are also studied as function of comonomer composition, as represented in Figure 5. The PPP triads are displayed on the left side of Figure 5 and they decrease as comonomer composition increases. A linear trend is observed for the different copolymers, fact that seems to indicate that polymerization temperature does not affect the final type of compositional sequences in the resulting materials. Thus, the catalytic system could play a significant role leading to similar type of triads, as can be deduced from PPP triads. This assumption can also be justified by other compositional triads represented in the right side of Figure 5 since linear tendencies are observed too.
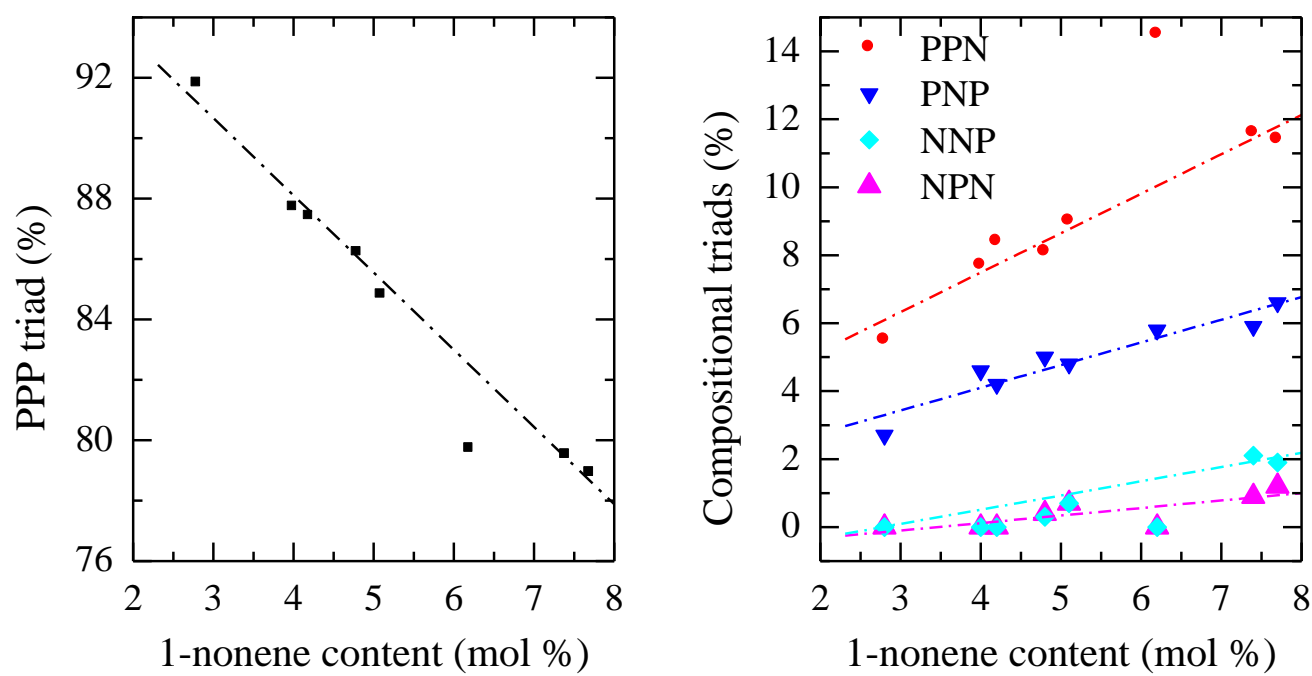

Figure 5. Composition triads as a function of comonomer content for the different propylene-co-1nonene copolymers.

The majority of the 1-nonene units are found as isolated entities, as deduced from the relationship between PPN and PNP. The PPN triads are close to twice the PNP sequences, indicating the mentioned isolation of the 1-nonene units, whilst a small proportion of PNN coexists, as can be deduced from Table 4, and also from the slopes of Figure 5. In addition, samples with low molecular weight could show the presence of 1 -nonene at the end of the polymeric chain.[62] This fact could explain the higher PPN slope found compared with that observed for PNP triads. Incorporation of 1-nonene comonomer could lead to a greater amount of termination reactions and, consequently, a higher number of PPN sequences. The lower slopes detected for the NPN and NNP triads can be easily justified because the low 1 -nonene feeding reduces the possibilities of this kind of triads in comparison with the higher proportion of propylene inside the reactor. 
These distinct molecular features exhibited by the different copolymers based on propylene and 1-nonene could lead to changes in the crystalline structure and thermal transitions. In order to analyze these variations, films were prepared by compression molding from the melt. Crystallization took place by applying a slow cooling from the molten state to room temperature. Resultant characteristics in terms of crystalline lattices and melting temperatures are evaluated by wide-angle $X$-ray diffraction (WAXD) and differential scanning calorimetry (DSC) measurements, respectively.

Figure 6 shows the X-ray profiles of the different copolymers under study, slowly crystallized from the melt. They are placed, from top to bottom, in order of increasing 1-nonene content, independently of the polymerization temperature used during synthesis.

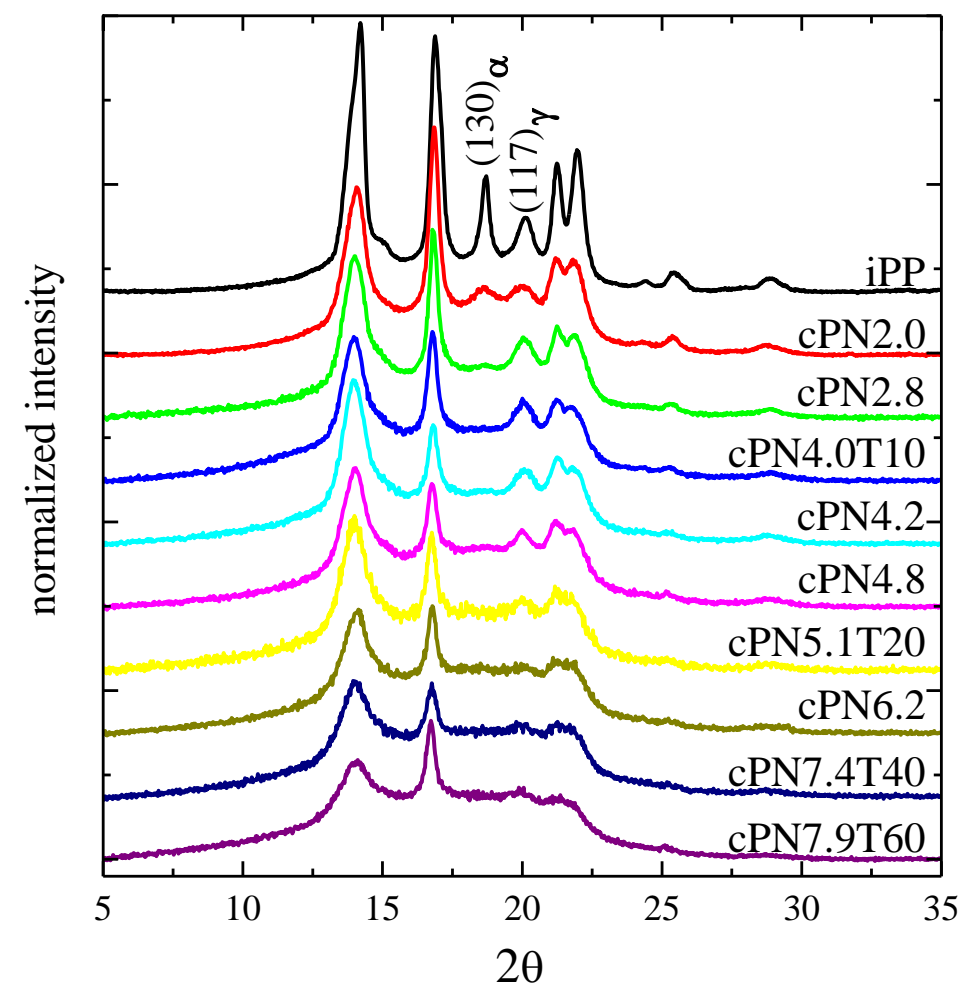

Figure 6. WAXD profiles at room temperature for all the copolymers synthesized. Samples are placed from top to bottom in order of increasing 1-nonene content, independently of polymerization temperature.

It is well known that IPP exhibits a very interesting polymorphic behavior, depending on the polymerization procedure, molecular weight, thermal history and use of different nucleants. Thus, three main crystalline lattices all sharing a three-fold conformation and labeled as $\alpha, \beta$ and $y$ have been reported, ${ }^{[63-66]}$ together with another phase of intermediate or mesomorphic order if a fast quenching is applied.[63,66-68] In 2005, a new polymorph was described, ${ }^{[15-22]}$ as mentioned in the Introduction, in 
copolymers with relatively large contents in 1-hexene or 1-pentene comonomers randomly distributed. And also the new $\varepsilon$ modification, reported in a highly stereodefective ${ }^{[2]}$ iPP sample.

The X-ray diffractograms in Figure 6 for these samples, after crystallization at slow rate, show the coexistence of two distinct crystalline polymorphs: monoclinic and orthorhombic crystals. ${ }^{[27,35]}$ Thus, their characteristics distinctive reflections, the (130) one for the monoclinic a crystallites at around $18.7^{\circ}$ and the (117) diffraction peak for the $y$ lattice at about $20.0^{\circ}$, are observed. Figure 6 depicts that the orthorhombic content is increasing rather appreciably in the copolymers. Thus, the homopolymer displays, approximately, $41 \%$ of such polymorph, and its content increases to $53 \%$ for cPN2.0 and to $93 \%$ for cPN2.8. At higher comonomer contents there is an almost complete absence of the monoclinic polymorph, as clearly deduced from those WAXD patterns. Consequently, these copolymers with propylene and 1-nonene crystallize mainly as orthorhombic crystals at low cooling rate. This fact is different to that described for propylene copolymers incorporating ethylene, 1-butene, 1-hexene, 1heptene and 1-octene crystallized at analogous slow rates or under isothermal conditions. $[25,27,35]$ The $y$ form has been found to be boosted in random copolymers of propylene and a-olefins at low crystallization rates $[25,27,35,63,64,66]$ and also in the case of low-molecular weight iPP.[69] The present copolymers, incorporating randomly distributed 1-nonene as comonomer, have been crystallized at around $0.5-1^{\circ} \mathrm{C} / \mathrm{min}$ and their molar mass is relatively low, all these features favoring the formation of orthorhombic crystals. In addition, this counit is longer than those aforementioned.

Figure 6 also displays, as expected, that intensity of the different diffractions is dependent on 1nonene content. The amount of comonomer reduces the degree of crystallinity but this decrease is somewhat minimized because crystallization has taken place under rather favorable conditions. If a fast cooling would be imposed the impact of the large length of 1-nonene would be much more important.[30]

The phase transitions among the different phases were studied by differential scanning calorimetry experiments at a heating rate of $10^{\circ} \mathrm{C} / \mathrm{min}$. In order of increasing temperatures, the glass transition is that one appearing at the lowest temperatures. On heating experiments, it is observed in these copolymers approximately in the range from $-20^{\circ}$ to $0^{\circ} \mathrm{C}$, the latter value for the homopolymer.

At higher temperatures, the melting processes are observed, as shown in Figure 7(a). Thus, several endothermic events take place from 20 to $140{ }^{\circ} \mathrm{C}$. A first process occurs at about $35-50{ }^{\circ} \mathrm{C}$, which is related to the annealing that crystallites of small size undergo because of their reorganization at room temperature after processing. ${ }^{[30,70,71]}$ It appears with very small intensity in the homopolymer, but its intensity increases rather appreciably for the copolymers, because the amount of smaller crystals increases at those large contents. 


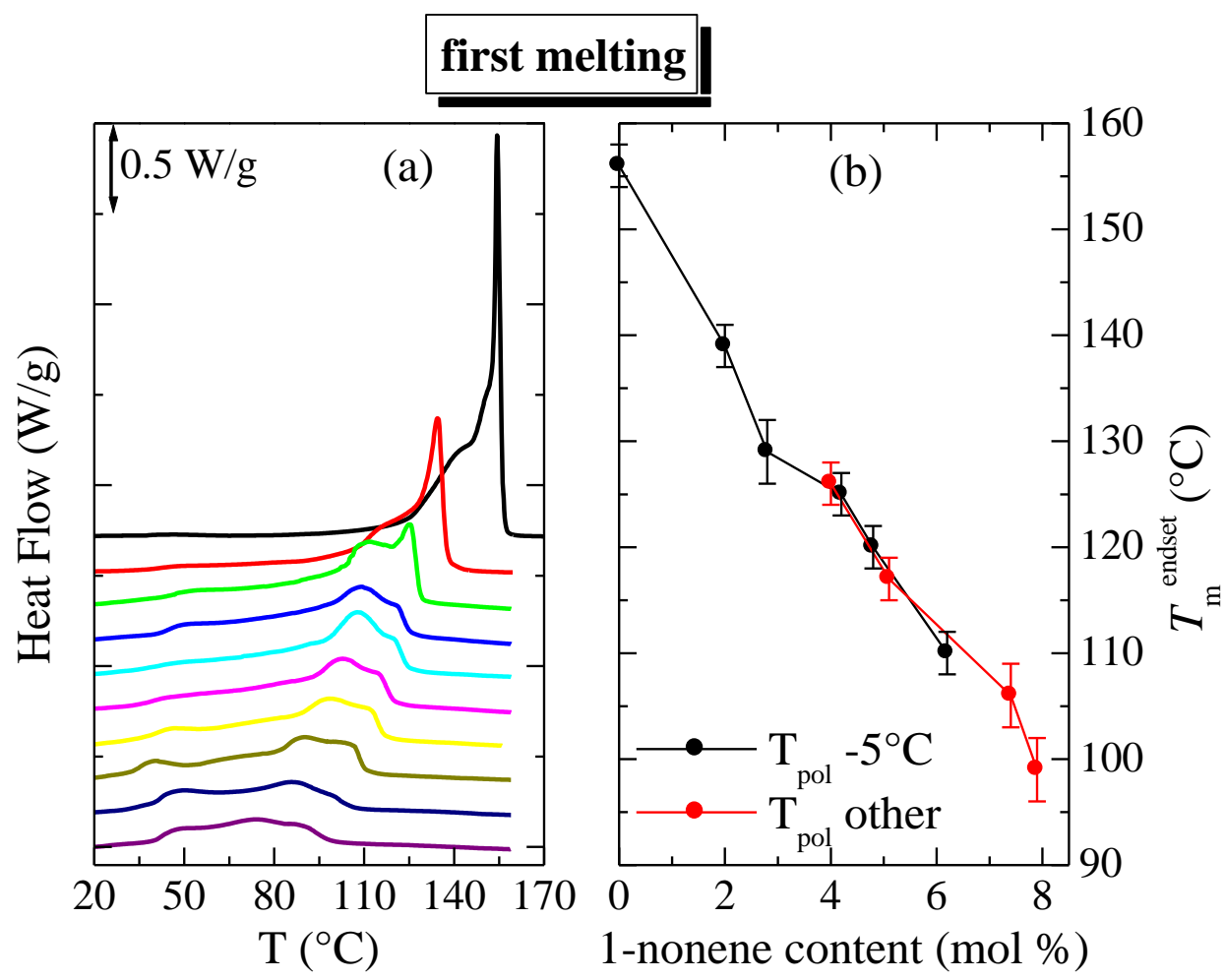

Figure 7. (a) Heat flow curves at $10^{\circ} \mathrm{C} / \mathrm{min}$ in the melting temperature interval during the first melting process (samples are placed as in Figure 6 in order of increasing 1-nonene content, independently of polymerization temperature); (b) dependence on 1-nonene content of the endset of melting temperature for the copolymers slowly crystallized during processing.

The main melting process, as observed in Figure $7(\mathrm{a})$, is composed by two overlapped endotherms. These two components could be ascribed to different causes. The first one can be related to coexistence of monoclinic and orthorhombic polymorphs. This is an expected feature in these propylene based copolymers slowly crystallized, as already commented. This fact would involve the observation of two merged phase transitions, the one at the lowest temperature associated with melting of the orthorhombic crystals and another ascribed to melting of the monoclinic entities at high temperatures. ${ }^{[72,73]}$ According to expectations, the high-temperature component should decrease as the comonomer content increases, since Figure 6 has shown that most of the crystallites for high comonomer contents are orthorhombic and only a minor amount are monoclinic ones.

Other reason leading to shapes of superimposed peaks could be the existence of meltingrecrystallization phenomena, which are expected to be more probable as the size of the initial crystals is small. When considering the results in Figures 6 and 7(a), it can be concluded, therefore, that the first reason (melting of the two polymorphs) is responsible at low comonomer contents for the two components in the melting endotherm, while melting-recrystallization phenomena are expected to be 
the cause in the case of high comonomer contents, where the amount of monoclinic crystals was found to be negligible.

Figure $7(b)$ represents the dependence of the endset melting temperature on 1-nonene composition. This endset temperature has been selected because of the aforementioned superposition of melting components. A fairly linear variation of that melting temperature with the comonomer content is observed for the samples synthesized at $-5{ }^{\circ} \mathrm{C}$. The rest of samples copolymerized at different temperatures follow also a linear variation, probably with a slightly different slope (although practically within the experimental error).

Figure 8 shows the results achieved during the subsequent crystallization process in the calorimetric experiments: the exothermic cooling curves (Figure 8(a)) and the dependence of peak crystallization temperature $\left(T_{c}\right)$ on 1-nonene content (Figure $\left.8(b)\right)$. The applied cooling rate was $10^{\circ} \mathrm{C} / \mathrm{min}$ in these calorimetric experiments, which is at least a decade faster than that imposed during film processing.

It has been described that calorimetric measurements at distinct cooling rates have allowed the formation of the iPP mesophase to be observed. Those required for iPP homopolymer and copolymers at low comonomer contents are ultra-fast rates ${ }^{[74]}$ (outside the range of conventional DSC) but the cooling rates needed decrease very much as either content or length of comonomer increase.[30,31,74] Accordingly, three different polymorphs can be developed depending on 1-nonene composition in the copolymers: orthorhombic, monoclinic and mesomorphic entities. At low comonomer contents, the former is less favored since cooling rate is now $10^{\circ} \mathrm{C} / \mathrm{min}$ and monoclinic crystal formation is boosted. Anyway, a unique narrow exotherm is observed during crystallization in Figure 8(a). An increase in the 1-nonene amount within the copolymers leads to a noticeable broadening of the crystallization process (see curve of dark yellow color, copolymer cPN6.2), which is much more evident for the exotherm of copolymer cPN7.4T40, where the observation of two exothermic components is clearly obvious. The appearance of these two overlapped processes can be ascribed to formation of the mesomorphic entities together with either the monoclinic or orthorhombic crystallites. The long length of this counit triggers mesophase development in spite of the relatively low rate used during the cooling run, i.e. $10^{\circ} \mathrm{C} / \mathrm{min}$. These mesomorphic entities will be majority in the copolymers with the highest contents, as deduced from the relative enlargement of the intensity of the low temperature peak exhibited by copolymers cPN7.4T40 and cPN7.9T60, represented by navy and purple colors, respectively. Previous results in other copolymers indicated that the low temperature component is due to the mesophase formation. $[25,28,31,74]$ 


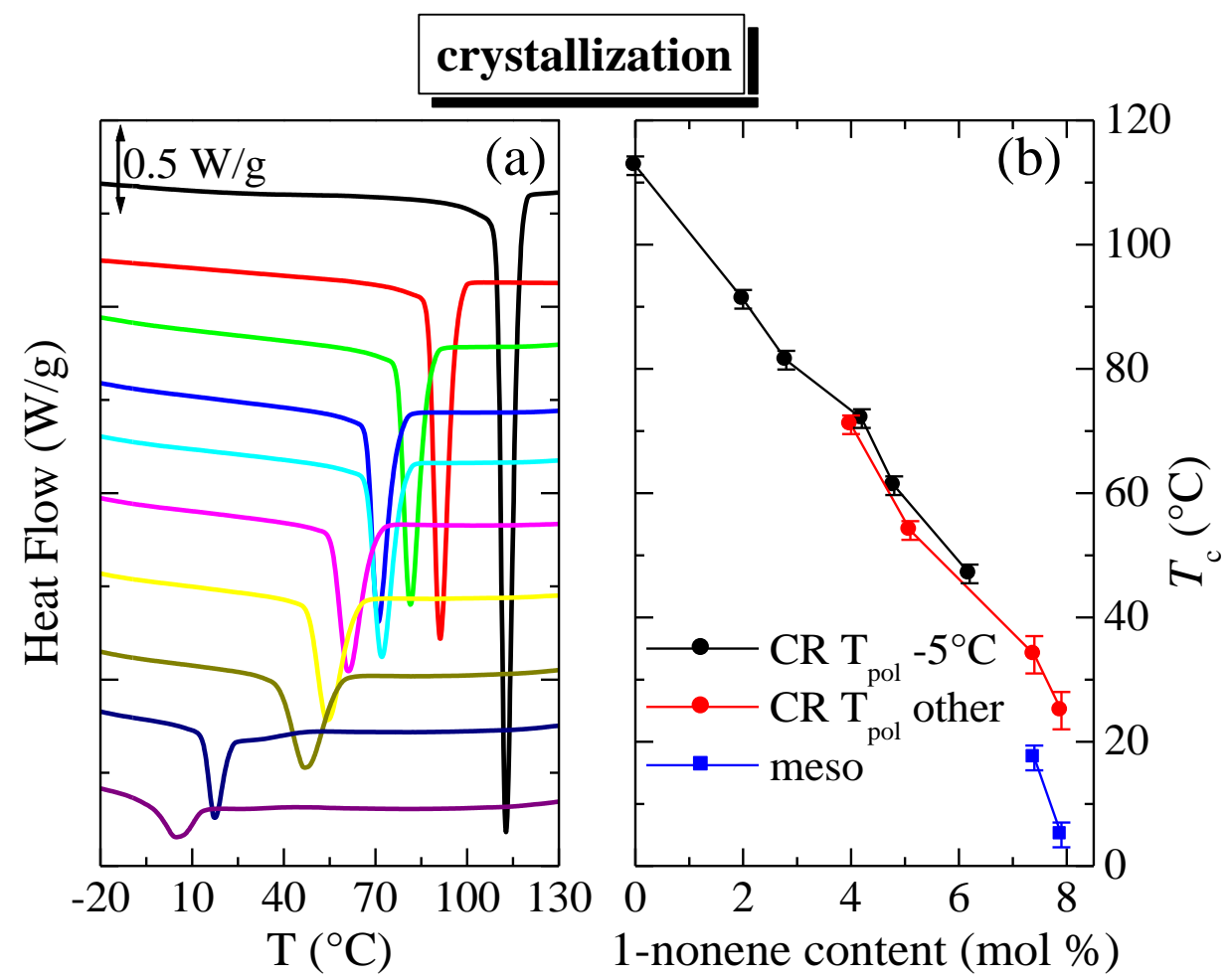

Figure 8. (a) Exothermic curves observed during cooling at $10^{\circ} \mathrm{C} / \mathrm{min}$ (samples are placed as in Figure

6 in order of increasing 1-nonene content, independently of polymerization temperature); (b) dependence on 1-nonene content of peak crystallization temperature and mesophase formation for the different copolymers under study.

Figure $8(b)$ shows the variation of peak crystallization temperature $\left(T_{c}\right)$ with 1-nonene content. As already observed for the melting temperature, a fairly linear variation with the comonomer composition is exhibited, probably with a slightly different slope as the polymerization temperature changes. An appropriate set of copolymers with different contents and prepared at the different temperatures would be needed to ascertain that slightly different variation, which, in fact, shall be expected when considering the microstructural changes aforementioned as function of the polymerization temperature. Defects in general have a profound influence on the crystallization behavior of iPP. ${ }^{775]}$

Moreover, and as mentioned above, two components are observed for the two copolymers with the highest contents, attributed to crystalline and mesophase, with the mesophase line appearing at around $20^{\circ} \mathrm{C}$ below that of the crystal.

By comparing Figures $7(b)$ and $8(b)$ it follows that the effect of comonomer content on the variation of the peak crystallization temperature (around $90{ }^{\circ} \mathrm{C}$ among the different copolymers) is considerably higher than that exhibited by the melting temperature, varying only around $60^{\circ} \mathrm{C}$. 
In order to ascertain the nature of the two exothermic components in samples with the two highest comonomer content, tentatively ascribed to true crystal and mesophase entities, real-time variable-temperature diffraction experiments with synchrotron radiation were performed on sample cPN7.9T60. In addition to the cooling rate used in the DSC experiments $\left(10^{\circ} \mathrm{C} / \mathrm{min}\right)$, two other rates, above and below that one, were tested: 60 and $3{ }^{\circ} \mathrm{C} / \mathrm{min}$. The corresponding diffractograms are shown in the upper part of Figure S1 in the Supporting Information, together with those corresponding to the subsequent melting experiments at $10^{\circ} \mathrm{C} / \mathrm{min}$ (lower plots in Figure S1). Due to the reduced crystallinity of this sample, the different features are much better observed in the pure ordered profiles, obtained by subtracting the corresponding amount of the amorphous component. Such profiles are shown in Figure 9 for the cooling (upper plots) and subsequent heating runs (lower representations).

First of all, it can be observed that only a small amount (see below) of ordered entities is developed under any condition, as corresponds to a copolymer with relatively high comonomer content. Furthermore, the behavior for the three cooling rates is rather different. Thus, cooling at $60^{\circ} \mathrm{C} / \mathrm{min}$ leads to a completely amorphous sample, which in the subsequent heating run (represented in the lower plot of Figure 9) first shows a cold "crystallization" into the mesophase, showing its two characteristic broad reflections, and this phase melts and recrystallizes later into monoclinic $\alpha$ crystals, before their final melting at around $90^{\circ} \mathrm{C}$.

In contrast, the monoclinic phase is the only one that is formed when cooling takes place at $3{ }^{\circ} \mathrm{C} / \mathrm{min}$, this phase being stable on further heating until its overall melting, which occurs again at around $90^{\circ} \mathrm{C}$.

An intermediate behavior is observed when cooling is performed at $10^{\circ} \mathrm{C} / \mathrm{min}$, with an initial formation of a very minor amount of monoclinic crystallites, starting at around $30^{\circ} \mathrm{C}$, followed by a much considerable growth of the mesophase at lower temperatures.

The amount of each of these two ordered phases at a given temperature can be estimated from those profiles. The procedure is not simple, owing to the overlapping of the different diffractions. It has been shown, however, that the area of the diffraction (040), which appears at $s$ values around $1.87 \mathrm{~nm}^{-1}$, represents an approximate amount of $13 \%$ in relation to the total area of the diffractogram for the monoclinic polymorph.[27,74] That diffraction is the one more isolated from the mesophase peaks and, then, the most appropriate reflection for the analysis. Subsequently, the proportion of mesophase is determined by subtraction of the amount of crystal from the total ordered area, which is directly determined from the profiles in Figure 9. 

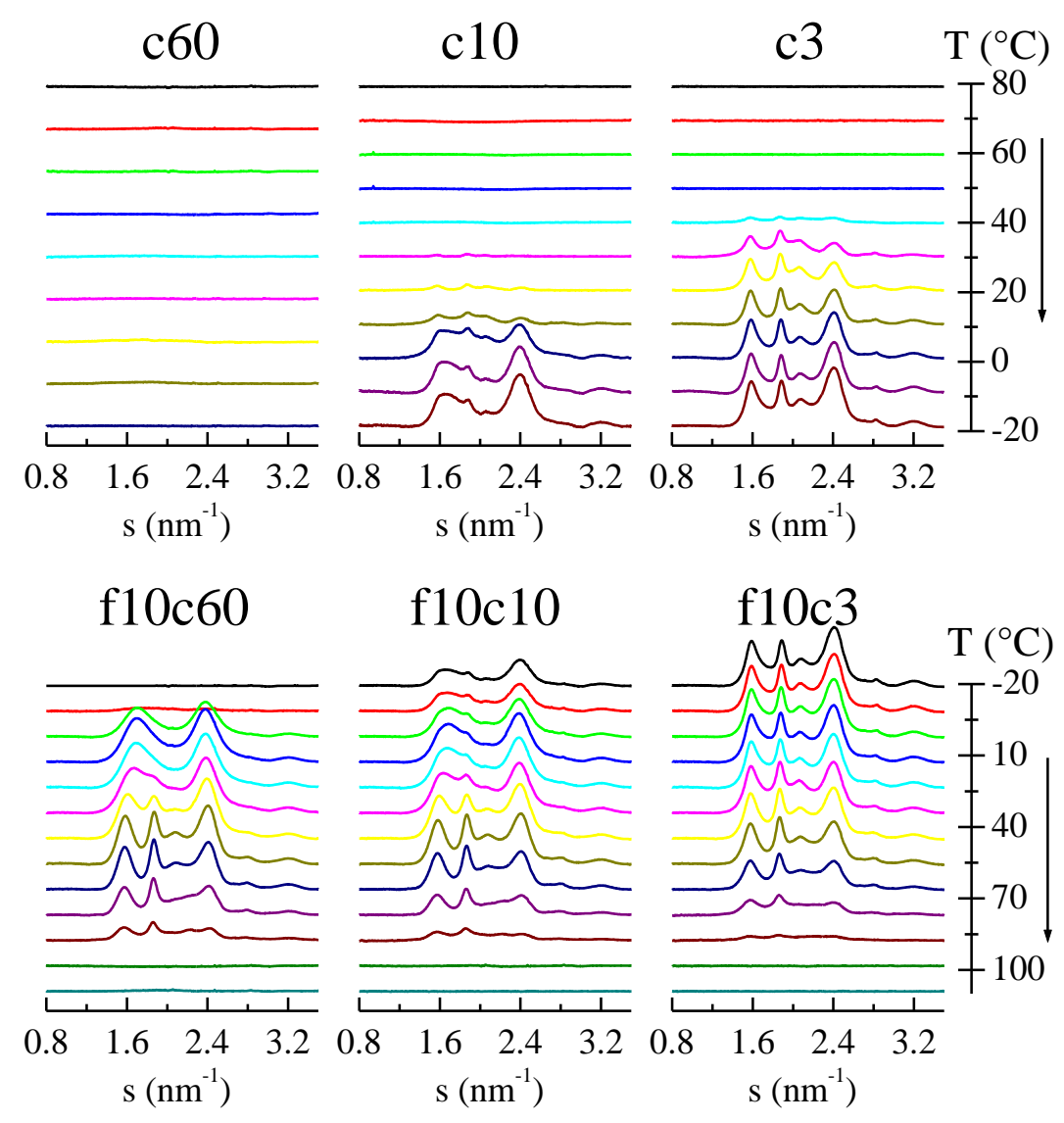

Figure 9. Profiles for the pure ordered component in sample cPN7.9T60, derived from the diffractograms represented in Figure S1 after appropriate subtraction of the amorphous component. Cooling from the melt at the indicated rates (upper plots) and subsequent melting experiments at $10^{\circ} \mathrm{C} / \mathrm{min}$ (lower plots). For clarity of presentation, only one every five diffractograms has been plotted.

With that approximation, and applying a rigorous smoothing to the original data, the estimated fractions of ordered components: total, monoclinic crystals $(\mathrm{CR})$ and mesophase, are shown for copolymer cPN7.9T60 in Figure 10 when it is cooled from the melt at $10{ }^{\circ} \mathrm{C} / \mathrm{min}$ and in the subsequent heating at identical rate. On cooling, it can be observed that exclusively monoclinic crystals are formed within the interval ranging from around 37 to $10^{\circ} \mathrm{C}$. These crystallites only represent a maximum crystallinity of about $3 \%$. At lower temperatures, the mesophase begins to form, reaching soon a maximum value of around $15 \%$ in the total amount of ordered phases, what renders a mesophase amount of $12 \%$.

The values achieved on heating, shown in the lower part of Figure 10, indicate that there is a small but appreciable increase of ordered phase at around $5{ }^{\circ} \mathrm{C}$, corresponding to an increase in the amount of mesophase. It appears that even at that relatively slow cooling rate of $10{ }^{\circ} \mathrm{C} / \mathrm{min}$, the sample does not "crystallize" at completion. Consequently, there is a small cold "crystallization" on heating. At 
higher temperatures, starting at around $20^{\circ} \mathrm{C}$, the mesophase melts and recrystallizes into monoclinic crystals without appreciable change in the total amount or ordered phase. That process seems not to be totally completed when the monoclinic crystals themselves begin to melt.

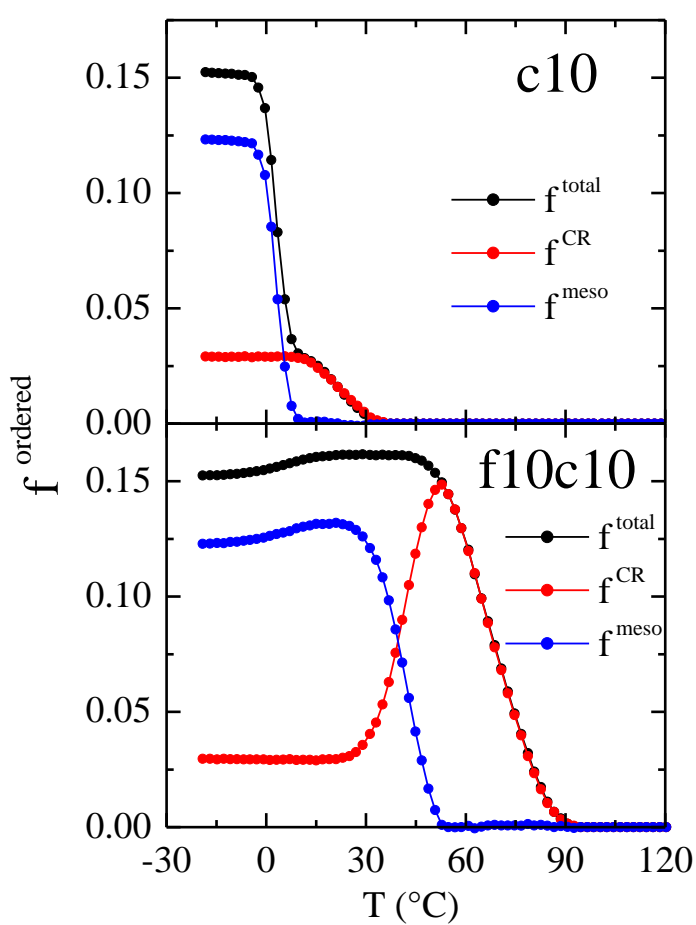

Figure 10. Estimated fractions of ordered components: total, monoclinic crystals (CR) and mesophase corresponding to copolymer cPN7.9T60 on the cooling from the melt at $10^{\circ} \mathrm{C} / \mathrm{min}$ (above) and on the subsequent heating at the same rate (below).

Derivatives of the phase contents represented in Figure 10, and those for the other two cooling rates, are compared in Figure 11 with the corresponding DSC curves, showing a rather good similarity. Thus, the synchrotron results almost perfectly reproduce the different DSC processes, but they have inestimable advantage of identifying the phases involved in the different processes from the corresponding diffractograms. Moreover, the melting-recrystallization of the mesophase, a process hardly noticeable in the melting DSC curves, is perfectly visualized in the derivative of the crystalline amount (f10c10CR).

The information deduced from these synchrotron experiments is, therefore, really valuable, especially for the identification and evolution of the mesophase (and polymorphism in general). The behavior for other compositions (and polymerization temperatures) is rather similar, although it has to be considered that the cooling rates needed for observing the mesophase (or the totally amorphous sample) are inversely proportional to the comonomer content. Thus, extremely high cooling rates, 
outside the usual range of conventional DSC or the present synchrotron experiments, are necessary at low comonomer contents, as mentioned above.
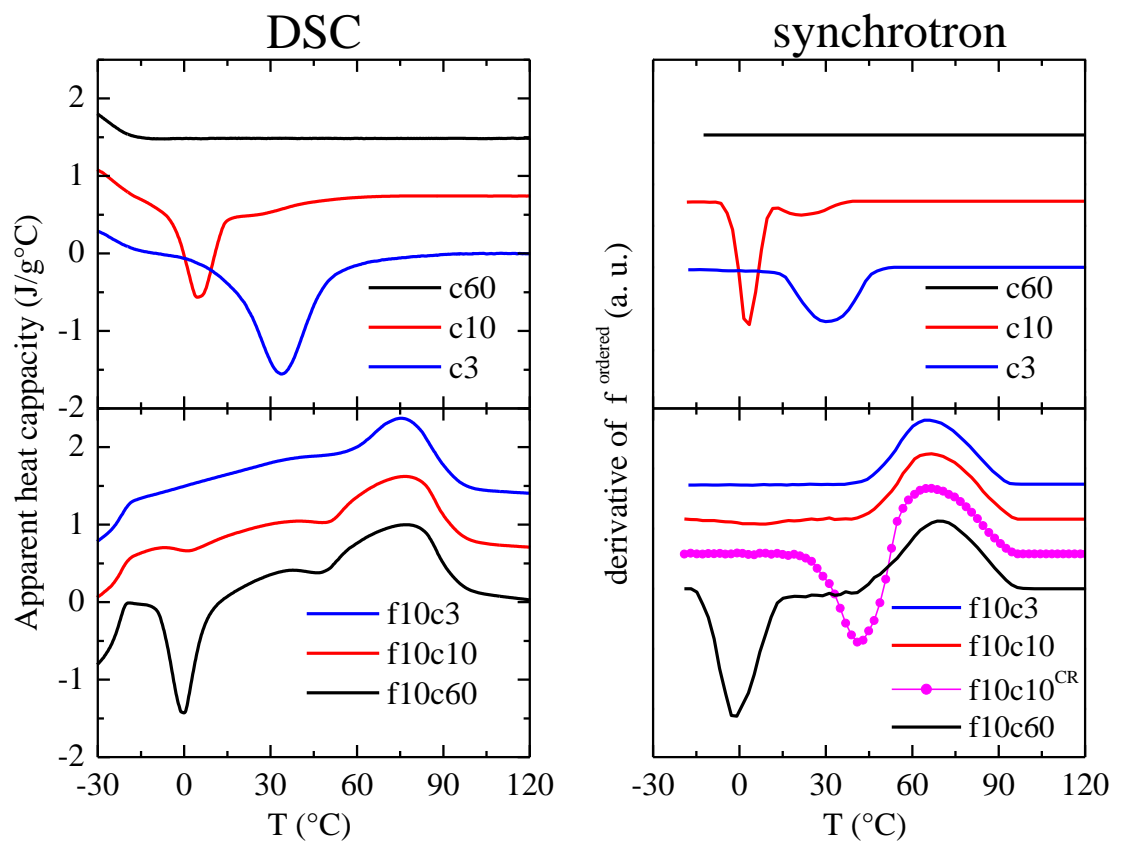

Figure 11. Left: Normalized cooling (top) and heating (bottom) DSC curves for copolymer cPN7.9T60 at the indicated rates. Right: Corresponding derivative of the total fraction of ordered phases determined in

the synchrotron experiments. The derivative of the crystalline amount ( $\left.110 \mathrm{c} 10^{\mathrm{CR}}\right)$ on heating after cooling at $10^{\circ} \mathrm{C} / \mathrm{min}$ is also shown in the right bottom frame.

A final aspect is concerned with the formation of the iPP mesophase and the role that it plays in the general ordering process of the polymer. As reported before ${ }^{[76]}$ and derived from these results, the mesophase of IPP is competing with the crystalline structures, contrary to the case of liquid crystalline mesophases, which are precursors of the eventual subsequent crystallization. Nevertheless, that iPP mesophase recrystallizes on melting into the monoclinic phase at common DSC rates, which has been also observed for the present copolymers with 1-nonene.

\section{Conclusions}

Copolymers based on propylene with 1-nonene as comonomer are satisfactorily synthesized using a metallocene catalyst and different temperatures. An increase in the polymerization temperature leads to both a rise of the catalytic activity and a significant decrease in isotacticity. In addition, this variable seems to play an important role promoting the incorporation of the comonomeric chains. 
The WAXD results derived from these copolymers slowly cooled from the melt indicate that crystallization leads mainly to the development of orthorhombic crystals. Thus, the homopolymer displays, approximately, a $41 \%$ of such polymorph, and the content increases to $53 \%$ for cPN2.0, to $93 \%$ for cPN2.8, and there is an almost complete absence of the monoclinic $\alpha$ polymorph at higher comonomer contents.

The main melting process in these samples is composed of two overlapped endotherms. They correspond to the melting of the orthorhombic and monoclinic polymorphs at low comonomer contents while melting-recrystallization phenomena seem to be the cause at high comonomer contents, where the amount of monoclinic crystals is negligible.

Dependences of both the endset melting temperature and crystallization temperature on 1nonene composition show a fairly linear variation for the samples polymerized at $-5{ }^{\circ} \mathrm{C}$. The rest of copolymers prepared at different temperatures also follow a linear variation, probably with a slightly different slope (although practically within the experimental error). Moreover, two components are noticeable in the DSC cooling curves for the two copolymers with the highest 1-nonene contents, assigned to crystal and mesophase formation, with the mesophase line appearing at around $20^{\circ} \mathrm{C}$ below that of the crystal.

Real-time variable-temperature diffraction experiments with synchrotron radiation performed on sample cPN7.9T60 allow determining the nature of those two exothermic contributions, crystal and mesophase, when cooling at $10{ }^{\circ} \mathrm{C} / \mathrm{min}$, as in the DSC experiments. The behavior observed at the other two cooling rates tested is, however, rather different. Thus, a completely amorphous sample is generated when cooling is performed at $60^{\circ} \mathrm{C} / \mathrm{min}$. This shows in the subsequent heating, firstly, a cold "crystallization" into the mesophase and, secondly, its melting and further recrystallization into monoclinic $\alpha$ crystals, which undergo their final melting at around $90{ }^{\circ} \mathrm{C}$. In contrast, monoclinic crystallites are the only ones developed when cooling is carried out at $3{ }^{\circ} \mathrm{C} / \mathrm{min}$, these crystals being stable on further heating until their total melting, which again takes place at around $90^{\circ} \mathrm{C}$.

Moreover, these synchrotron measurements allow estimating the amount of each of the two ordered phases (crystal and mesophase) as a function of temperature. The derivative of those phase contents shows a rather good similarity with the corresponding DSC curves, although these X-ray diffraction profiles involve the inestimable advantage of showing the phases existing in the different processes. Furthermore, the melting-recrystallization of the mesophase, process barely identified in the melting DSC curves, is perfectly visualized in the derivative of the crystalline amount. 


\section{Acknowledgements}

The financial support obtained from project MAT2016-79869-C2-1-P (AEI/FEDER, UE) is greatly acknowledged. The synchrotron experiments were performed at beamline BL11-NCD-SWEET at ALBA Synchrotron Light Facility with the collaboration of ALBA staff.

\section{References}

[1] A. Rahimi, J.M. García, Nature Reviews Chemistry 2017, 1, 0046.

[2] F.E. Dierberg, N.E. Carriker, Environ. Sci. Techn. 1994, 28, 16-25.

[3] D.S. Scotp, P. Majerski, J. Piskorz, D. Radlein, M. Barnickel, Canadian J. Chem. Eng. 1999, 77, 1021-1027.

[4] A. Miller, Environ. Sci. Techn. 1994, 28, 16 A.

[5] T.M. Kruse, H.-W. Wong, L.J. Broadbelt, Macromolecules 2003, 36, 9594-9607.

[6] M. Stürzel, S. Mihan, R. Mülhaupt, Chem. Reviews 2016, 116, 1398-1433.

[7] A. Deep, D. Bhatt, V. Shrivastav, S.K. Bhardwaj, P. Malik, In Synthesis, Characterization and Applications of Polyolefin Based Eco-Friendly Polymer Composites, T.S. Inamuddin, M.R. Kumar, A. Asiri, Eds., Springer: Cham, 2019, pp 65-103.

[8] R.N. Kumar, R. Nagarajan, F. Chee Fun, P. Leng Seng, Eur. Polym. J. 2000, 36, 2491-2497.

[9] A. Prasetya, L. Liu, J. Litster, F. Watanabe, K. Mitsutani, G.H. Ko, Chem. Eng. Sci. 1999, 54, 3263-3271.

[10] H. Sinn, W. Kaminisky, Organomet. Chem. 1980, 18, 99-149.

[11] F.R.W.P. Wild, L. Zsolnai, G. Huttner, H.H. Brintzinger, J. Organomet. Chem. 1982, 232, 233-247.

[12] J.A. Ewen, J. Am. Chem. Soc. 1984, 106, 6355-6364.

[13] W. Kaminsky, K. Kulper, H.H. Brintzinger, F.R.W.P. Wild, Angew. Chem., Int. Ed. 1985, 24, 507-508.

[14] E.T. Kiesewetter, S. Randoll, M. Radlauer, R.M. Waymouth, J. Am. Chem. Soc. 2010, 132, 5566-5567.

[15] B. Poon, M. Rogunova, A. Hiltner, E. Baer, S.P. Chum, A. Galeski, E. Piorkowska, Macromolecules 2005, 38, 1232-1243.

[16] C. De Rosa, F. Auriemma, P. Corradini, O. Tarallo, S. Dello lacono, E. Ciaccia, L. Resconi, J. Am. Chem. Soc. 2006, 128, 80-81.

[17] B. Lotz, J. Ruan, A. Thierry, G.C. Alfonso, A. Hiltner, E. Baer, E. Piorkowska, A. Galeski, Macromolecules 2006, 39, 5777-5781.

[18] C. De Rosa, S. Dello lacono, F. Auriemma, E. Ciaccia, L. Resconi, Macromolecules 2006, 39, 6098-6109.

[19] C. De Rosa, F. Auriemma, G. Talarico, O.R. de Ballesteros, Macromolecules 2007, 40, 8531-8532.

[20] C. De Rosa, O.R. Ballesteros, F. Auriemma, M.R. Di Caprio, Macromolecules 2012, 45, 2749-2763.

[21] E. Pérez, M.L. Cerrada, R. Benavente, J.M. Gómez-Elvira, Macromol. Res. 2011, 19, 1179-1185.

[22] A. García-Peñas, J.M. Gómez-Elvira, V. Lorenzo, E. Pérez, M.L. Cerrada, Polymer 2017, 130, 17-25.

[23] L. Boragno, P. Stagnaro, F. Forlini, F. Azzurri, G.C. Alfonso, Polymer 2013, 54, 1656-1662.

[24] A. García-Peñas, J.M. Gómez-Elvira, E. Pérez, M.L. Cerrada, J. Polym. Sci., Part A: Polym. Chem. 2013, $51,3251-3259$.

[25] A. García-Peñas, J.M. Gómez-Elvira, M.L. Cerrada, E. Pérez, RSC Adv. 2016, 6, 82907-82915.

[26] A. García-Peñas, J.M. Gómez-Elvira, R. Barranco-García, E. Pérez, M.L Cerrada, Polymer 2016, 99, 112121.

[27] A. García-Peñas, J.M. Gómez-Elvira, V. Lorenzo, E. Pérez, M.L. Cerrada, Eur. Polym. J. 2015, 64, 52-61. 
[28] A. García-Peñas, J.M. Gómez-Elvira, M.U. de la Orden, M.L. Cerrada, E. Pérez, Polym. Int. 2016, 65, 596604.

[29] H. Palza, J.M. López-Majada, R. Quijada, J.M. Pereña, R. Benavente, E. Pérez, M.L. Cerrada, Macromol. Chem. Phys. 2008, 209, 2259-2267.

[30] M.J. Polo-Corpa, R. Benavente, T. Velilla, R. Quijada, E. Pérez, M.L. Cerrada, Eur. Polym. J. 2010, 46, 1345-1354.

[31] J. Arranz-Andrés, R. Parrilla, M.L. Cerrada, E. Pérez, Macromolecules 2013, 46, 8557-8568.

[32] B. Lotz, Macromolecules 2014, 47, 7612-7624.

[33] C. De Rosa, F. Auriemma, O. Ruiz de Ballesteros, L. Resconi, I. Camurati, Macromolecules 2007, 40, $6600-6616$.

[34] J. Arranz-Andrés, I. Suárez, B. Peña, R. Benavente, E. Pérez, M.L. Cerrada, Macromol. Chem. Phys. 2007, 208, 1510-1521.

[35] K. Jeon, H. Palza, R. Quijada, R.G. Alamo, Polymer 2009, 50, 832-844.

[36] D. Mileva, D. Cavallo, L. Gardella, G.C. Alfonso, G. Portale, L. Balzano, R. Androsch, Polym. Bull. 2011, 67, 497-510.

[37] M.C. Sacchi, F. Forlini, S. Losio, I. Tritto, G. Costa, P. Stagnaro, I. Tincul, U.M. Wahner, Macromol. Symp. 2004, 213, 57-68.

[38] I. Tincul, D. Joubert, R. Sanderson, Macromol. Symp. 2007, 260, 58-64.

[39] M.L. Cerrada, R. Benavente, E. Pérez, J. Mater. Res. 2001, 16, 1103-1111.

[40] R. Barranco-García, A.E. Ferreira, M.R. Ribeiro, V. Lorenzo, A. García-Peñas, J.M. Gómez-Elvira, E. Pérez, M.L. Cerrada, Polymer 2018, 151, 218-230.

[41] K. Heiland, W. Kaminsky, Makromol. Chem. 1992, 193, 601-610.

[42] R. Quijada, G.B. Galland, R.S. Mauler, Macromol. Chem. Phys. 1996, 197, 3091-3098.

[43] W. Kaminsky, H. Hahnsen, In Advances in Polyolefins, R. B. Seymour, T. Cheng, Eds., Plenum Press: New York, 1987, p. 361.

[44] A. Akimoto, A. Yano, In Metallocene-Based Polyolefins, J. Scheirs, W. Kaminsky, Eds., John Wiley \& Sons, Chichester, 2000, p. 287.

[45] T. Tsutsui, N. Kashiwa, Polym. Commun. 1988, 29, 180-183.

[46] J.C.W. Chien, T. Nozaki, J. Polym. Sci., Part A: Polym. Chem. 1993, 31, 227-237.

[47] M. A. Da Silva, G. B Galland, J. Polym. Sci., Part A: Polym. Chem. 2008, 46, 947-957.

[48] F. Forlini, I. Tritto, P. Locatelli, M.C. Sacchi, F. Piemontesi, Macromol. Chem. Phys. 2000, 201, 401-408.

[49] H.F. Hermann, L.L. Böhm, Polym. Commun. 1991, 32, 58-61.

[50] J. Koivumäki, J.V. Seppälä, Macromolecules 1993, 26, 5535-5538.

[51] A. García-Peñas, J.M. Gómez-Elvira, E. Pérez, M.L. Cerrada, J. Polym. Sci., Part A: Polym. Chem. 2014, $52,2537-2547$.

[52] K. Li, L. Wu, Molecules 2017, 22, 751.

[53] Z. Cai, N. Yuushou, T. Shiono, Proceedings of the First Asian Polyolefin Workshop. Collection: Studies in Surf. Sci. Catal. 2006, 161, 189-192.

[54] V. Volkis, E. Smolensky, A. Lisovskii, M.S. Eisen, J. Polym. Sci., Part A: Polym. Chem. 2005, 43, 45054516.

[55] L. Resconi, I. Camurati, O. Sudmeijer, Topics In Catalysis 1999, 7, 145-163.

[56] V. Busico, R. Cipullo, N. Friederichs, H. Linssen, A. Segre, V. Van Axel Castelli, G. van der Velden, Macromolecules 2005, 38, 6988-6996.

[57] Q. Wu, A. García-Peñas, R. Barranco-García, M.L. Cerrada, R. Benavente, E. Pérez, J.M. Gómez-Elvira, Polymers 2019, 11, 1266.

[58] G. Natta, I. Pasquon, A. Zambelli, G. Gatti, J. Polym. Sci. 1961, 51, 387-398.

[59] Y.V. Kissin, A.J. Brandolini, Macromolecules 1991, 24, 2632-2633. 
[60] U.M. Wahner, I. Tincul, D.J. Joubert, E.R. Sadiku, F. Forlini, S. Losio, I. Tritto, M.C. Sacchi, Macromol. Chem. Phys. 2003, 204, 1738-1746.

[61] F.F.N. Escher, G.B. Galland, M. Ferreira, J. Polym. Sci., Part A: Polym. Chem. 2003, 41, 2531-2541.

[62] A. García-Peñas, C. Martínez, M.L. Cerrada, E. Pérez, J.M. Gómez-Elvira. J. Polym. Sci., Part A: Polym. Chem. 2017, 55, 843-854.

[63] S. Brückner, S.V. Meille, V. Petraccone, B. Pirozzi, Prog. Polym. Sci. 1991, 16, 361-404.

[64] B. Lotz, J.C. Wittmann, A.J. Lovinger, Polymer 1996, 37, 4979-4992.

[65] J. Varga, J. Mater. Sci. 1992, 27, 2557-2579.

[66] P.J. Phillips, K. Mezghani, In The Polymeric Materials Encyclopedia, J.C. Salamone Ed., CRC Press: Boca Raton, 1996. Vol. 9, p. 6637.

[67] P. Corradini, C. de Rosa, G. Guerra, V. Petraccone, Polym. Commun. 1989, 30, 281-285.

[68] J. Arranz-Andrés, R. Benavente, E. Pérez, M.L. Cerrada, Polym. J. 2003, 35, 766-777.

[69] S.V. Meille, S. Brückner, Nature 1989, 340, 455-457.

[70] S. Bensason, J. Minick, A. Moet, S. Chum, A. Hiltner, E. Baer, J. Polym. Sci., Part B: Polym. Phys. 1996, 34, 1301-1315.

[71] A. Alizadeh, L. Richardson, J. Xu, S. McCartney, H. Marand, Y.W. Cheung, S. Chum, Macromolecules 1999, $32,6221-6235$.

[72] I.L. Hosier, R.G. Alamo, P. Esteso, J.R. Isasi, L. Mandelkern, Macromolecules 2003, 36, 5623-5636.

[73] R. Barranco-García, J.M. López-Majada, J.C. Martínez, J.M. Gómez-Elvira, E. Pérez, M.L. Cerrada, Micropor. Mesopor. Mat. 2018, 272, 209-216.

[74] E. Pérez, J.M. Gómez-Elvira, R. Benavente, M.L. Cerrada, Macromolecules 2012, 45, 6481-6490.

[75] D. Tranchida, L. Resconi, Polymer Crystallization 2018, e10022.

[76] E. Pérez, M.L. Cerrada, A. García-Peñas, J.M. Gómez-Elvira, J.P. Fernández-Blázquez, A. MartínezGómez, D. López-Velázquez, J. C. Martínez, Eur. Polym. J. 2016, 81, 661-673. 